\title{
Study of Academician Leadership Type and Innovational Approach
}

\author{
Serkan Zengin ${ }^{1, *}$, Mahmut Gülle ${ }^{2}$, Erdi Kaya ${ }^{3}$ \\ ${ }^{1}$ School of Physical Education and Sport, Ağrı İbrahim Çeçen University, Ağrı, Turkey \\ ${ }^{2}$ School of Physical Education and Sport, Mustafa Kemal University, Hatay, Turkey \\ ${ }^{3}$ Faculty of Sport Sciences, Akdeniz University, Antalya, Turkey
}

Copyright $\bigcirc 2018$ by authors, all rights reserved. Authors agree that this article remains permanently open access under the terms of the Creative Commons Attribution License 4.0 International License

\begin{abstract}
The purpose of this study is to examine whether there is any difference in the leadership and innovation approaches of the academicians who teach in different fields in terms of gender, if doing sports, title, seniority, administrative duty and marital status variables. The information form developed by the researcher in this research and the Leadership Styles and Innovation Approach inventory developed by Eysenck and Eysenck in 1975 and adapted to Turkish by Bayram in 2013 were used [3]. The sample of the study consists of 232 male and 33 female academicians from Mustafa Kemal University working in different branches of education selected by using random sampling method. Non-parametric analysis methods have been preferred because of the distributions of the obtained data. Participants' gender, marital status, regular sports activities, academic title, administrative duties and occupational seniority were found to have significant differences in terms of scale sub-dimensions in terms of all demographic values. When looking at the correlation values of the study, it is also found that there are significant relationships among the sub-dimensions.
\end{abstract}

Keywords Leader, Innovation, Academic Leadership

\section{Introduction}

Many people believe that leadership is an inherent superiority. It should not be forgotten that some innate characteristics; some skills such as leadership, entrepreneurship or a fighting spirit play only a role in helping to become a leader. It is necessary to pay attention to these personality traits that the people who come to leadership position have in order to guarantee the power and authority in their hands is used correctly and effectively. However, there are a number of leadership qualities that people cannot have innately. These characteristics can be learned, acquired and regularly developed [2]. Leadership is an important phenomenon for the effectiveness of organizations [32].

Defining the concept of leadership in a wide variety of forms in fact shows that it is difficult to define as a comprehensive and generally accepted definition of this concept [7]. This naturally comes from the perspective and conditions of people or societies. However, the qualities and features that should be in a leader can be determined within a general framework, even with outlines; the way in which the leader behaves the strategy to be followed, his aims and goals [26]. In short, leadership should be governed by the organizational spirit [1], effectively, adequately and dynamically, taking into account the personality traits of organizational values, norms and ideals while making the organization more effective.

The concept of leadership plays an active role in the development process of humanity: the development of the industry, the social values, the progress of education and the development of technology. Because of the increase of the human population and the competition environment, the understanding of leadership has developed rapidly. As a result of these developments, we see that leadership types are emerging in order to ensure that competitiveness is well managed and that qualified people who will enable people to work correctly and steadily are brought to the community. In addition to being an effective role of education institutions in attracting qualified people, our educators are seen as important role models for students with their academic personalities and visionary structure.

Recently, while teacher-centered teaching is widely seen in research on education provided by educators, it emphasizes the need to bring the domains of the educators beyond the classroom and into school-wide leadership activities. Foreseeing the role of the educators, including comprehensive thinking and use of quality training activities [11], every school has an educator who is a leader or has a potential for being a leader. If the educators are supported by their leadership, if the school and students learn how to make use of Unclear what this refers to this 
resource will be more useful. Many educators want to do their best for their students. Politicians, managers and other leaders outside the classroom seem to have the responsibility of educators to create realistic dialogue with leaders and to find the resources they need to succeed [21].

The leadership role of the education director has been grouped under nine headings [22]. They are: Demonstrating... Demonstrating that academic achievement can be achieved, Preparing the environment for high expectations, Acting like an education leader, Being strong and dynamic, Effective exchange of views with teachers, Discipline and order, Using resources in the best possible way, Using time well, Evaluation of results [22].

Global change makes human and education more valuable. Education is seen as a factor in sustaining prosperity in rich countries, while in the poor countries is seen as driving force for development. As economic equilibrium, social deterioration and technological developments increase, demands on educational systems are expected to increase in the positive direction [4]. The benevolence of the citizens, the integration of the social components of the country, the durability of the economy and the long-term national security will be based on the effectiveness of schools and education [15]. Reformers all over the world say they need new innovations in their children's current education. The growing global economic competition and the increasing demand for technological demands of information societies are driving an innovative structure of education models. Politicians who want to strengthen their economic competitiveness place more emphasis on innovative education systems in order to develop the education and skills that will be successful in the global economy [19]. Innovative methods of teaching and learning are the student-centered or constructivist methods. The tasks assigned to the educators here should play a role in providing active participation by leading the students with the teaching. This way, students will look at their own solutions to question themselves and make sense of their future [18]. During the review of Turkish literature, empirical studies on the management and leadership of academics are quite limited and there is no study on the effects of these tasks on the professional development of academicians [17], [10], [23], [13], [25].

In short; managers, administrators, teachers, academicians etc. who are seen as the leaders of education are seen as epic leaders in the minds of the students as they all play an important role in the school environment. If we want to leave: I suggest replace with productive as educators, we must lead the students to become innovative and gain strategic thinking skills.

\section{Method and Methods}

\subsection{Research Model}

In the research, a method for descriptive survey and relational survey aimed at revealing the current situation has been used.

Descriptive survey is a research approach aimed at describing models in the past or as they exist. The individual or object under investigation is tried to be defined as it is within its own conditions. No attempt is made to alter or influence them in any way. Relational survey models are research models aiming to determine the presence and/or degree of exchange between two or more variables [16].

\subsection{Population and Sampling}

The population of the research is composed of academicians who work in Mustafa Kemal University in different branches of education in the 2016-2017 academic year. The academicians participating in the study were selected by chance sampling method. The sample group of the population consists of 232 male and 33 female participants.

\subsection{Data Collection Tools}

\section{Leadership Styles and Innovation Approach Scale Information}

The data collection tool consists of 48 items in five-point Likert scale in addition to demographic questions. The 48-item scale was developed by Bayram [3]. The scale is 1 $=$ strongly disagree and $5=$ strongly agree. The first 33 items are based on leadership styles and the last 15 on innovation approaches. Scores of the scale subscales were calculated in accordance with the factor analyzes at the source of the scale. In leadership styles scale, items 1-10-21-22-27 measure autocratic leadership, items 13-15-23-32 measure democratic leadership, items 19-20-25-26-28-33 measure humanist leadership, items 6-8-11-16-24-30 measure bureaucratic leadership, and Items 3-4-5-12 measure charismatic leadership. 8 questions in the leadership scale were not included in this study as it is not in the scale's source. In the scale items 34-35-36-41-43-44-46 measure the innovation approaches and 8 items related to innovation approaches not used in the scale source are not evaluated in this study.

\subsection{Statistical Analysis}

SPSS 22 program was used in the analysis of the obtained data. Because scale scores did not show normal distribution, non-parametric analysis methods were preferred in comparison of demographic variables and scale scores in examining the relationship between scale scores. The Mann Whitney U test was used to compare the scale scores according to gender, marital status and regular sports activities of the participants. The Kruskal Wallis $\mathrm{H}$ test was used to compare the scale scores according to the academic title, administrative duties and occupational seniority of the participants. If there is a significant 
difference between the groups in the result of Kruskal Wallis H test, Mann Whitney U test was used as a post hoc test to determine the difference between groups. The Spearman Correlation analysis was used to examine the relationship between leadership and innovation scales. Significance level was accepted as $p<0,05$ in all analyzes.

\section{Findings}

$12.5 \%$ of the participants were women and $87.5 \%$ were men. $21.5 \%$ of the participants were instructors, $43.8 \%$ were assistant professor, $22,6 \%$ were associate professors and $12,1 \%$ were professors, $3 \%$ were deans / directors, $15,8 \%$ assistant dean/manager and $81.1 \%$ of them were department heads. $7,2 \%$ of the participants were single and $92,8 \%$ were married. $5.7 \%$ of the survey participants had 1-5 years of occupational seniority, $6.4 \%$ had $6-10$ years, $27.5 \%$ had $11-15$ years, $21.5 \%$ had $16-20$ years and $38.9 \%$ had $20+$ years of occupational seniority.

When the table 2 is examined, it appeared that the participants had a moderate level of autocratic, bureaucratic and charismatic leadership styles and a high level of democratic and humanist leadership styles. In addition, the level of innovation approaches of participants was moderate.

Table 1. Descriptive Statistics on Participants' Demographic Information

\begin{tabular}{|c|c|c|c|}
\hline Variable & Sub-variables & f & $\%$ \\
\hline \multirow{2}{*}{ Gender } & Women & 33 & 12,5 \\
\hline & Men & 232 & 87,5 \\
\hline \multirow{4}{*}{ Title } & Instructor & 57 & 21,5 \\
\hline & Asst. Prof. & 116 & 43,8 \\
\hline & Docent & 60 & 22,6 \\
\hline & Professor & 32 & 12,1 \\
\hline \multirow{3}{*}{ Administrative duties } & Dean / Director & 8 & 3,0 \\
\hline & Asst. Dean/Director & 42 & 15,8 \\
\hline & Head of Department & 215 & 81,1 \\
\hline \multirow{2}{*}{ Marital status } & Single & 19 & 7,2 \\
\hline & Married & 246 & 92,8 \\
\hline \multirow{5}{*}{ Professional seniority } & $1-5$ years & 15 & 5,7 \\
\hline & 6-10 years & 17 & 6,4 \\
\hline & $11-15$ years & 73 & 27,5 \\
\hline & 16-20 years & 57 & 21,5 \\
\hline & $20+$ years & 103 & 38,9 \\
\hline \multirow{2}{*}{ Regular sporting situation } & Yes & 101 & 38,1 \\
\hline & No & 164 & 61,9 \\
\hline
\end{tabular}

Table 2. Descriptive Statistics on Participants' Leadership Styles and Innovation Approaches

\begin{tabular}{c|c|c|c|c}
\hline Scale & Sub-dimensions & N & X & Ss \\
\hline \multirow{4}{*}{ Leadership } & Autocratic & 265 & 4,02 &, 512 \\
\cline { 2 - 5 } & Democratic & 265 & 4,38 &, 440 \\
\cline { 2 - 5 } & Humanist & 265 & 4,33 &, 509 \\
\cline { 2 - 5 } & Bureaucratic & 265 & 4,14 &, 433 \\
\cline { 2 - 5 } & Charismatic & 265 & 3,75 &, 503 \\
\hline \multirow{2}{*}{ Innovation } & Innovation & 265 & 4,10 & \\
\hline
\end{tabular}


When the table 3 was examined, it could be seen that the participants' leadership style, autocratic, democratic and charismatic leadership levels and levels of innovation approach did not differ statistically by gender $(p>0,05)$, that the humanist leadership and bureaucratic leadership levels differed statistically according to gender $(p<0,05)$. These two leadership styles seemed to have a higher level of female participants.

When the table 4 was examined, it could be seen that the participants did not differ statistically by the leadership styles of the autocratic, democratic, bureaucratic and charismatic leadership levels ( $p>0,05)$. It also could be seen that humanist leadership levels and innovation approach levels differed statistically according to their titles $(\mathrm{p}<0,05)$. In the humanist leadership style, it seemed that this leadership style was higher for assistant professors and associate professors than instructors. In the innovation approach, it could be seen that the participants who are assistant professors had this approach at a higher level than the participants who are instructors and docent.

Table 3. Comparison of Leadership Styles and Innovation Approaches by Gender of Participants

\begin{tabular}{|c|c|c|c|c|c|c|c|c|}
\hline Sub-dimensions & Cinsiyet & $\mathbf{N}$ & $\mathbf{X}$ & Ss & Mean & Total & $\mathbf{U}$ & $\mathbf{p}$ \\
\hline \multirow{2}{*}{$\begin{array}{c}\text { Autocratic } \\
\text { (Leadership) }\end{array}$} & Women & 33 & 3,92 &, 531 & 132,29 & 4365,5 & \multirow{2}{*}{3804,5} & \multirow{2}{*}{,954 } \\
\hline & Men & 232 & 4,03 &, 509 & 133,10 & 30879,5 & & \\
\hline \multirow{2}{*}{$\begin{array}{l}\text { Democratic } \\
\text { (Leadership) }\end{array}$} & Women & 33 & 4,35 &, 364 & 130,23 & 4297,5 & \multirow{2}{*}{3736,5} & \multirow{2}{*}{, 821} \\
\hline & Men & 232 & 4,38 &, 450 & 133,39 & 30947,5 & & \\
\hline \multirow{2}{*}{$\begin{array}{c}\text { Humanist } \\
\text { (Leadership) }\end{array}$} & Women & 33 & 4,15 &, 335 & 93,15 & 3074,0 & \multirow{2}{*}{2513,0} & \multirow{2}{*}{, 001} \\
\hline & Men & 232 & 4,35 &, 525 & 138,67 & 32171,0 & & \\
\hline \multirow{2}{*}{$\begin{array}{l}\text { Bureaucratic } \\
\text { (Leadership) }\end{array}$} & Women & 33 & 3,76 &, 317 & 66,12 & 2182,0 & \multirow{2}{*}{1621,0} & \multirow{2}{*}{, 000} \\
\hline & Men & 232 & 4,19 &, 422 & 142,51 & 33063,0 & & \\
\hline \multirow{2}{*}{$\begin{array}{l}\text { Charismatic } \\
\text { (Leadership) }\end{array}$} & Women & 33 & 3,86 &, 307 & 136,80 & 4514,5 & \multirow{2}{*}{3702,5} & \multirow{2}{*}{, 756} \\
\hline & Men & 232 & 3,73 & ,623 & 132,46 & 30730,5 & & \\
\hline \multirow{2}{*}{$\begin{array}{c}\text { Innovation } \\
\text { approach }\end{array}$} & Women & 33 & 4,08 &, 355 & 124,36 & 4104,0 & \multirow{2}{*}{3543,0} & \multirow{2}{*}{,485 } \\
\hline & Men & 232 & 4,10 &, 521 & 124,36 & 4104,0 & & \\
\hline
\end{tabular}

Table 4. Comparison of Leadership Styles and Innovation Approaches by Participants' Academic Title

\begin{tabular}{|c|c|c|c|c|c|c|c|c|}
\hline Sub-dimensions & Title & $\mathbf{N}$ & $\mathbf{X}$ & Ss & Mean & $\mathbf{x} 2$ & $\mathbf{p}$ & $\begin{array}{c}\text { Differences } \\
\text { between groups }\end{array}$ \\
\hline \multirow{4}{*}{$\begin{array}{c}\text { Autocratic } \\
\text { (Leadership) }\end{array}$} & Instructor & 57 & 3,98 & ,475 & 128,70 & \multirow{4}{*}{2,851} & \multirow{4}{*}{, 415} & \multirow[t]{4}{*}{ 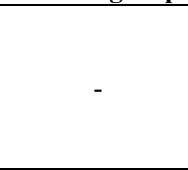 } \\
\hline & Asst. Prof. & 116 & 4,08 & ,576 & 141,44 & & & \\
\hline & Docent & 60 & 3,94 & ,406 & 122,46 & & & \\
\hline & Professor & 32 & 4,00 & ,505 & 129,84 & & & \\
\hline \multirow{4}{*}{$\begin{array}{l}\text { Democratic } \\
\text { (Leadership) }\end{array}$} & Instructor & 57 & 4,38 & ,431 & 135,80 & \multirow{4}{*}{2,262} & \multirow{4}{*}{, 520} & \multirow{4}{*}{-} \\
\hline & Asst. Prof. & 116 & 4,39 & ,493 & 139,02 & & & \\
\hline & Docent & 60 & 4,36 & ,327 & 124,48 & & & \\
\hline & Professor & 32 & 4,35 & ,457 & 122,16 & & & \\
\hline \multirow{4}{*}{$\begin{array}{c}\text { Humanist } \\
\text { (Leadership) }\end{array}$} & Instructor & 57 & 4,19 & ,448 & 103,18 & \multirow{4}{*}{12,265} & \multirow{4}{*}{, 007} & \multirow{4}{*}{$1<2,1<3$} \\
\hline & Asst. Prof. & 116 & 4,37 & ,588 & 145,90 & & & \\
\hline & Docent & 60 & 4,37 & ,419 & 136,61 & & & \\
\hline & Professor & 32 & 4,35 & ,425 & 132,58 & & & \\
\hline \multirow{4}{*}{$\begin{array}{l}\text { Bureaucratic } \\
\text { (Leadership) }\end{array}$} & Instructor & 57 & 4,09 & ,387 & 126,74 & \multirow{4}{*}{5,819} & \multirow{4}{*}{, 121} & \multirow{4}{*}{ - } \\
\hline & Asst. Prof. & 116 & 4,13 & ,467 & 130,78 & & & \\
\hline & Docent & 60 & 4,09 &, 387 & 127,27 & & & \\
\hline & Professor & 32 & 4,30 & ,449 & 162,97 & & & \\
\hline \multirow{4}{*}{$\begin{array}{l}\text { Charismatic } \\
\text { (Leadership) }\end{array}$} & Instructor & 57 & 3,78 &, 610 & 134,87 & \multirow{4}{*}{7,638} & \multirow{4}{*}{, 054} & \multirow{4}{*}{-} \\
\hline & Asst. Prof. & 116 & 3,78 & ,597 & 139,90 & & & \\
\hline & Docent & 60 & 3,82 & ,393 & 136,03 & & & \\
\hline & Professor & 32 & 3,45 & ,781 & 98,98 & & & \\
\hline \multirow{4}{*}{$\begin{array}{l}\text { Innovation } \\
\text { approach }\end{array}$} & Instructor & 57 & 4,02 & ,445 & 112,69 & \multirow{4}{*}{10,407} & \multirow{4}{*}{,015 } & \multirow{4}{*}{$1<2,2>3$} \\
\hline & Asst. Prof. & 116 & 4,17 & ,545 & 148,27 & & & \\
\hline & Docent & 60 & 4,05 & ,434 & 120,73 & & & \\
\hline & Professor & 32 & 4,11 & ,550 & 136,83 & & & \\
\hline
\end{tabular}


When the table 5 was examined, it was found that the participants' humanist leadership style did not differ statistically according to the administrative duties $(\mathrm{p}>0,05)$, the autocratic, democratic, bureaucratic and charismatic leadership levels and the level of innovation approach were statistically different according to the administrative duties $(p<0,05)$. Autocratic and charismatic leadership styles and participants who are deans / directors in the innovation approach seemed to have these features at a higher level than asst. deans / directors and department heads. In the demographic leadership style, participants who are deans seemed to have this feature at a higher level than asst. deans / directors and department heads, and asst. deans / directors had this feature at a higher level than participants who are head of departments. In the bureaucratic leadership, participants who are deans and those department heads seemed to have this leadership style at a higher level than asst. deans / directors.

When the table 6 was examined, it was found that the levels of humanist and charismatic leadership styles did not differ statistically by marital status $(p>0,05)$. Autocratic, democratic, bureaucratic leadership levels and innovation approach levels are statistically different according to their marital status $(p<0,05)$. It is seen that married participants had a higher level of these characteristics with significant differences.

Table 5. Comparison of Leadership Styles and Innovation Approaches by Participants' Administrative Duties

\begin{tabular}{|c|c|c|c|c|c|c|c|c|}
\hline Sub-dimensions & Administrative duty & $\mathbf{N}$ & $\mathbf{X}$ & Ss & Sira ort. & $\mathbf{x} 2$ & $\mathbf{p}$ & $\begin{array}{c}\begin{array}{c}\text { Gruplar arası } \\
\text { farklar }\end{array} \\
\end{array}$ \\
\hline \multirow{3}{*}{$\begin{array}{c}\text { Autocratic } \\
\text { (Leadership) }\end{array}$} & Dean / Director & 8 & 4,50 &, 355 & 203,88 & \multirow{3}{*}{9,682} & \multirow{3}{*}{, 008} & \multirow{3}{*}{$1>2,1>3$} \\
\hline & $\begin{array}{c}\text { Asst. Dean / } \\
\text { Director }\end{array}$ & 42 & 4,11 & ,412 & 147,43 & & & \\
\hline & Head of Depart. & 215 & 3,98 &, 524 & 127,54 & & & \\
\hline \multirow{3}{*}{$\begin{array}{c}\text { Democratic } \\
\text { (Leadership) }\end{array}$} & Dean / Director & 8 & 4,81 & ,347 & 208,38 & \multirow{3}{*}{12,502} & \multirow{3}{*}{, 002} & \multirow{3}{*}{$1>2,1>3,2>3$} \\
\hline & $\begin{array}{c}\text { Asst. Dean / } \\
\text { Director }\end{array}$ & 42 & 4,43 & ,436 & 152,38 & & & \\
\hline & Head of Depart. & 215 & 4,35 & ,436 & 126,41 & & & \\
\hline \multirow{3}{*}{$\begin{array}{c}\text { Humanist } \\
\text { (Leadership) }\end{array}$} & Dean / Director & 8 & 4,21 & , 194 & 96,75 & \multirow{3}{*}{2,784} & \multirow{3}{*}{,249 } & \multirow{3}{*}{-} \\
\hline & $\begin{array}{c}\text { Asst. Dean / } \\
\text { Director }\end{array}$ & 42 & 4,23 &, 542 & 123,93 & & & \\
\hline & Head of Depart. & 215 & 4,35 & ,509 & 136,12 & & & \\
\hline \multirow{3}{*}{$\begin{array}{l}\text { Bureaucratic } \\
\text { (Leadership) }\end{array}$} & Dean / Director & 8 & 4,42 & 089 & 190,25 & \multirow{3}{*}{9,041} & \multirow{3}{*}{, 011} & \multirow{3}{*}{$1>2,2<3$} \\
\hline & $\begin{array}{c}\text { Asst. Dean / } \\
\text { Director }\end{array}$ & 42 & 3,98 & ,400 & 108,88 & & & \\
\hline & Head of Depart. & 215 & 4,16 & ,439 & 135,58 & & & \\
\hline \multirow{3}{*}{$\begin{array}{l}\text { Charismatic } \\
\text { (Leadership) }\end{array}$} & Dean / Director & 8 & 4,50 & ,779 & 208,38 & \multirow{3}{*}{9,365} & \multirow{3}{*}{, 009} & \multirow{3}{*}{$1>2,1>3$} \\
\hline & $\begin{array}{l}\text { Asst. Dean / } \\
\text { Director }\end{array}$ & 42 & 3,92 & ,352 & 141,79 & & & \\
\hline & Head of Depart. & 215 & 3,69 & 601 & 128,48 & & & \\
\hline \multirow{3}{*}{ Innovation approach } & Dean / Director & 8 & 4,68 & ,437 & 210,38 & 8,591 & ,014 & $1>2,1>3$ \\
\hline & $\begin{array}{c}\text { Asst. Dean / } \\
\text { Director }\end{array}$ & 42 & 4,06 & ,471 & 128,60 & & & \\
\hline & Head of Depart. & 215 & 4,09 &, 500 & 130,98 & & & \\
\hline
\end{tabular}

Table 6. Comparison of Leadership Styles and Innovation Approaches by Participants' Marital Status

\begin{tabular}{|c|c|c|c|c|c|c|c|c|}
\hline Sub-dimensions & $\begin{array}{l}\text { Marital } \\
\text { Status }\end{array}$ & $\mathbf{N}$ & $\mathbf{X}$ & Ss & Mean & Total & $\mathbf{U}$ & $\mathbf{p}$ \\
\hline \multirow{2}{*}{$\begin{array}{c}\text { Autocratic } \\
\text { (Leadership) }\end{array}$} & Single & 19 & 3,83 & ,293 & 93,58 & 1778,0 & \multirow{2}{*}{1588,0} & \multirow{2}{*}{,018 } \\
\hline & Married & 246 & 4,03 &, 523 & 136,04 & 33467,0 & & \\
\hline \multirow{2}{*}{$\begin{array}{c}\text { Democratic } \\
\text { (Leadership) }\end{array}$} & Single & 19 & 4,09 &, 253 & 68,97 & 1310,5 & \multirow{2}{*}{1120,5} & \multirow{2}{*}{,000 } \\
\hline & Married & 246 & 4,40 & ,444 & 137,95 & 33934,5 & & \\
\hline \multirow{2}{*}{$\begin{array}{c}\text { Humanist } \\
\text { (Leadership) }\end{array}$} & Single & 19 & 4,32 & ,368 & 127,37 & 2420,0 & \multirow{2}{*}{2230,0} & \multirow{2}{*}{, 737} \\
\hline & Married & 246 & 4,33 & ,519 & 133,43 & 32825,0 & & \\
\hline \multirow{2}{*}{$\begin{array}{l}\text { Bureaucratic } \\
\text { (Leadership) }\end{array}$} & Single & 19 & 3,83 & ,319 & 83,92 & 1594,5 & \multirow{2}{*}{1404,5} & \multirow{2}{*}{, 003} \\
\hline & Married & 246 & 4,16 & ,433 & 136,79 & 33650,5 & & \\
\hline \multirow{2}{*}{$\begin{array}{l}\text { Charismatic } \\
\text { (Leadership) }\end{array}$} & Single & 19 & 3,93 & ,201 & 150,89 & 2867,0 & \multirow{2}{*}{1997,0} & \multirow{2}{*}{,282 } \\
\hline & Married & 246 & 3,73 & ,612 & 131,62 & 32378,0 & & \\
\hline \multirow{2}{*}{$\begin{array}{l}\text { Innovation } \\
\text { approach }\end{array}$} & Single & 19 & 3,97 &, 380 & 95,05 & 1806,0 & \multirow{2}{*}{1616,0} & \multirow{2}{*}{,024 } \\
\hline & Married & 246 & 4,11 &, 510 & 135,93 & 33439,0 & & \\
\hline
\end{tabular}


When the table 7 is examined, it can be seen that the levels of autocratic, democratic and humanist leadership and innovation approach of the participants' leadership styles do not differ statistically according to regular sporting situation of participants $(p>0,05)$. The level of bureaucratic and charismatic leadership differed statistically according to the regular sporting situation $(p<0,05)$. Participants without regular sports had a higher level of bureaucratic leadership style, while those with regular sports had a higher level of charismatic leadership style.

When the table 8 is examined, it is seen that the participants' autocratic and democratic leadership levels do not differ statistically according to their vocational seniority $(p>0,05)$. The levels of humanist, bureaucratic and charismatic leadership and innovation approach differ statistically ( $<<0,05)$ according to occupational seniority. In the humanist leadership style, the participants with vocational seniority of 6-10 years, $11-15$ years, $16-20$ years and $20+$ years had a higher level than participants with vocational seniority of 1-5 years, while the participants with vocational seniority 16-20 years had this leadership style higher than those with 6-10 years, 11-15 years and $20+$ years. In the bureaucratic leadership style, the participants with occupational seniority of 6-10 years,
11-15 years, 16-20 years, and 20+ years had a higher level than the participants with occupational seniority of 1-5 years, while the participants with $20+$ years of vocational seniority had a higher level than those with vocational seniority of 6-10 years, $11-15$ years and 16-20 years. In the charismatic leadership style, the participants who have vocational seniority of 6-10 years had higher levels than those who had $1-5$ years, $6-10$ years, $11-15$ years and $16-20$ years. Participants with $11-15$ years, $16-20$ years and $20+$ years of vocational seniority in the innovation approach had a higher level than participants with 1-5 years, while participants with vocational seniority of 11-15 years and $20+$ years had a higher level than participants with 6-10 years.

When the table 9 was examined, it was found that there was a significant positive and moderate relationship between the participants' levels of democratic leadership and innovation approaches $(p<0,05)$. There was a positive and moderately significant relationship between levels of autocratic and humanist leadership and innovation approaches $(\mathrm{p}<0,05)$. , and a positive and moderate relation between bureaucratic leadership levels and innovation approach levels $(p<0,05)$. There was also a positive and low level of significant relationship between charismatic leadership levels and innovation approach levels $(p<0,05)$.

Table 7. Comparison of Leadership Styles and Innovation Approaches by Regular Sports Situations of Participants

\begin{tabular}{|c|c|c|c|c|c|c|c|c|}
\hline Sub-dimensions & $\begin{array}{c}\text { Regular } \\
\text { sports }\end{array}$ & $\mathbf{N}$ & $\mathbf{X}$ & Ss & Mean & Total & $\mathbf{U}$ & $\mathbf{p}$ \\
\hline \multirow{2}{*}{$\begin{array}{c}\text { Autocratic } \\
\text { (Leadership) }\end{array}$} & Yes & 101 & 4,00 & ,358 & 128,55 & 12983,5 & \multirow{2}{*}{7832,5} & \multirow[b]{2}{*}{,452 } \\
\hline & No & 164 & 4,02 &, 589 & 135,74 & 22261,5 & & \\
\hline \multirow{2}{*}{$\begin{array}{c}\text { Democratic } \\
\text { (Leadership) }\end{array}$} & Yes & 101 & 4,35 &, 376 & 130,05 & 13135,5 & \multirow{2}{*}{7984,5} & \multirow{2}{*}{,616 } \\
\hline & No & 164 & 4,39 & ,476 & 134,81 & 22109,5 & & \\
\hline \multirow{2}{*}{$\begin{array}{c}\text { Humanist } \\
\text { (Leadership) }\end{array}$} & Yes & 101 & 4,38 & ,419 & 138,44 & 13982,5 & \multirow{2}{*}{7732,5} & \multirow{2}{*}{,360 } \\
\hline & No & 164 & 4,29 & ,556 & 129,65 & 21262,5 & & \\
\hline \multirow{2}{*}{$\begin{array}{l}\text { Bureaucratic } \\
\text { (Leadership) }\end{array}$} & Yes & 101 & 4,03 & ,256 & 111,41 & 11252,0 & \multirow{2}{*}{6101,0} & \multirow{2}{*}{, 000} \\
\hline & No & 164 & 4,20 & ,504 & 146,30 & 23993,0 & & \\
\hline \multirow{2}{*}{$\begin{array}{l}\text { Charismatic } \\
\text { (Leadership) }\end{array}$} & Yes & 101 & 3,88 & ,493 & 152,43 & 15395,0 & \multirow{2}{*}{6320,0} & \multirow{2}{*}{,001 } \\
\hline & No & 164 & 3,67 & ,635 & 121,04 & 19850,0 & & \\
\hline \multirow{2}{*}{$\begin{array}{c}\text { Innovation } \\
\text { approach }\end{array}$} & Yes & 101 & 4,12 & ,288 & 130,84 & 13214,5 & \multirow{2}{*}{8063,5} & \multirow{2}{*}{,716 } \\
\hline & No & 164 & 4,09 & ,598 & 134,33 & 22030,5 & & \\
\hline
\end{tabular}


Table 8. Comparison of Leadership Styles and Innovation Approaches by Participants' Vocational Seniority

\begin{tabular}{|c|c|c|c|c|c|c|c|c|}
\hline Sub-dimensions & $\begin{array}{c}\text { Vocational } \\
\text { seniority }\end{array}$ & $\mathbf{N}$ & $\mathbf{X}$ & Ss & Mean & $\mathbf{x} 2$ & $\mathbf{p}$ & $\begin{array}{c}\text { Differences } \\
\text { between groups }\end{array}$ \\
\hline \multirow{5}{*}{$\begin{array}{c}\text { Autocratic } \\
\text { (Leadership) }\end{array}$} & $1-5$ years & 15 & 3,96 & ,304 & 128,50 & \multirow{5}{*}{5,908} & \multirow{5}{*}{,206 } & \multirow{5}{*}{-} \\
\hline & 6-10 years & 17 & 3,96 & ,257 & 108,41 & & & \\
\hline & 11-15 year & 73 & 4,01 & ,605 & 139,78 & & & \\
\hline & 16-20 year & 57 & 4,11 & ,285 & 147,90 & & & \\
\hline & $20+$ years & 103 & 3,98 & ,588 & 124,66 & & & \\
\hline \multirow{5}{*}{$\begin{array}{c}\text { Democratic } \\
\text { (Leadership) }\end{array}$} & 1-5 years & 15 & 4,15 & ,761 & 130,00 & \multirow{5}{*}{4,816} & \multirow{5}{*}{307} & \multirow{5}{*}{-} \\
\hline & 6-10 years & 17 & 4,25 & ,306 & 105,71 & & & \\
\hline & 11-15 year & 73 & 4,43 & ,453 & 146,30 & & & \\
\hline & 16-20 year & 57 & 4,38 & ,310 & 131,34 & & & \\
\hline & $20+$ years & 103 & 4,39 & ,444 & 129,43 & & & \\
\hline \multirow{5}{*}{$\begin{array}{c}\text { Humanist } \\
\text { (Leadership) }\end{array}$} & 1-5 years & 15 & 3,60 & ,930 & 67,10 & \multirow{5}{*}{51,736} & \multirow{5}{*}{, 000} & \multirow{5}{*}{$\begin{array}{c}1<2,1<3,1<4, \\
1<5,2<4,3<4, \\
4>5\end{array}$} \\
\hline & 6-10 years & 17 & 4,46 & ,280 & 149,91 & & & \\
\hline & 11-15 year & 73 & 4,25 & ,430 & 117,45 & & & \\
\hline & 16-20 year & 57 & 4,66 & ,265 & 190,77 & & & \\
\hline & $20+$ years & 103 & 4,28 &, 470 & 118,85 & & & \\
\hline \multirow{5}{*}{$\begin{array}{l}\text { Bureaucratic } \\
\text { (Leadership) }\end{array}$} & $1-5$ years & 15 & 3,70 &, 169 & 46,80 & \multirow{5}{*}{33,771} & \multirow{5}{*}{, 000} & \multirow{5}{*}{$\begin{array}{c}1<2,1<3,1<4, \\
1<5,2<5,3<5, \\
4<5\end{array}$} \\
\hline & 6-10 years & 17 & 3,97 & ,319 & 115,44 & & & \\
\hline & 11-15 year & 73 & 4,07 & ,456 & 124,63 & & & \\
\hline & 16-20 year & 57 & 4,12 & ,394 & 124,88 & & & \\
\hline & $20+$ years & 103 & 4,28 & ,421 & 158,88 & & & \\
\hline \multirow{5}{*}{$\begin{array}{l}\text { Charismatic } \\
\text { (Leadership) }\end{array}$} & $1-5$ years & 15 & 3,80 &, 254 & 130,90 & \multirow{5}{*}{13,748} & \multirow{5}{*}{, 008} & \multirow{5}{*}{$\begin{array}{c}1<2,2>3,2>4 \\
2>5\end{array}$} \\
\hline & 6-10 years & 17 & 4,21 & ,377 & 191,00 & & & \\
\hline & 11-15 year & 73 & 3,76 & ,521 & 127,78 & & & \\
\hline & 16-20 year & 57 & 3,83 & ,444 & 143,12 & & & \\
\hline & $20+$ years & 103 & 3,61 &, 724 & 121,83 & & & \\
\hline \multirow{5}{*}{$\begin{array}{l}\text { Innovation } \\
\text { approach }\end{array}$} & $1-5$ years & 15 & 3,51 & ,797 & 76,70 & \multirow{5}{*}{21,379} & \multirow{5}{*}{, 000} & \multirow{5}{*}{$\begin{array}{c}1<3,1<4,1<5 \\
2<3,2<5\end{array}$} \\
\hline & 6-10 years & 17 & 3,92 & ,332 & 79,03 & & & \\
\hline & 11-15 year & 73 & 4,13 & ,472 & 131,96 & & & \\
\hline & $16-20$ year & 57 & 4,03 &, 516 & 136,87 & & & \\
\hline & $20+$ years & 103 & 4,23 & ,412 & 148,70 & & & \\
\hline
\end{tabular}

Table 9. An Analysis of the Relationship between Participants' Leadership Styles and Innovation Approaches

\begin{tabular}{c|c|c|c|c|c|c}
\hline \multicolumn{2}{c|}{} & $\begin{array}{c}\text { Autocratic } \\
\text { (Leadership) }\end{array}$ & $\begin{array}{c}\text { Democratic } \\
\text { (Leadership) }\end{array}$ & $\begin{array}{c}\text { Humanist } \\
\text { (Leadership) }\end{array}$ & $\begin{array}{c}\text { Bureaucratic } \\
\text { (Leadership) }\end{array}$ & $\begin{array}{c}\text { Charismatic } \\
\text { (Leadership) }\end{array}$ \\
\hline \multirow{2}{*}{$\begin{array}{c}\text { Innovation } \\
\text { approach }\end{array}$} & $\mathbf{r}$ &, 449 &, 722 &, 473 &, 388 &, 168 \\
\cline { 2 - 7 } & $\mathbf{p}$ &, 000 &, 000 &, 000 &, 000 &, 006 \\
\hline
\end{tabular}

\section{Discussion and Conclusions}

Innovative Approaches, Autocratic, Democratic, Humanist, Bureaucratic and Charismatic Leadership styles of the academicians participating in the study are discussed in this section by looking at the results obtained from the scale scores according to gender, marital status, regular sports, academic titles, administrative duties and vocational seniority. Managers in educational institutions, especially higher education institutions, need to have academic and managerial leadership qualities [31]. Mentoring help people better manage their career opportunities by providing a role model or as a friend, regardless of their leadership style. And thus provide them with psychosocial counseling that allows them to develop their own leadership skills [20].

While the level of autocratic, democratic and charismatic leadership and innovation approach did not 
differ statistically according to the gender of the participants, when the level of humanist leadership and bureaucratic leadership was examined, it was seen that statistically significant difference was found in favor of the female participants. Different culture structures may occur when the organizational culture is considered in business environments [5]. For example, it can be expected that a man or woman can have a dominant culture [6]. These cultural differences reveal different values and reveal the most important formation and difference in the organization [5], [29]. It can be said that because of the difference in gender, women in our country do not have a hard time expressing themselves, and that people provide opportunities for humanistic and bureaucratic approach for women. The average leadership behavior differs by sex and marital status group. The determination of the leadership behavior of women is lower than that of men [33].

The participants' autocratic, democratic, bureaucratic and charismatic leadership levels did not differ significantly, but humanist leadership levels and levels of innovation approach differed statistically significantly according to their titles. In the humanist leadership style, it seemed that the instructors had a higher level than assistant professors and docents. As one of the main differences that led to such an understanding, it can be inferred that that the workload of the academicians (course hours) and the dialogue gates with the students were kept active and balanced. The academicians in the humanist position collect and theorize biographies and experiences of leaders and administrators. It treats notable leadership practices and knowledge of high-ranking people in a story-like and biographical context [24]. In the innovation approach, it is seen that the participants who are assistant professors have this approach at a higher level than the participants who are instructors and docents. Due to the academic promotion system that exists in our country, need for trying to put forth very serious researches especially for the academicians with the title of assistant professor can cause the increase of innovative approaches.

It was observed that participants' humanist leadership style levels did not differ statistically according to the administrative duties, but the levels of autocratic, democratic, bureaucratic and charismatic leadership and innovation approaches differed statistically according to the administrative duties. Deans/directors seemed to have features showing autocratic and charismatic leadership styles and innovation approaches at a higher level than assistant deans / directors and department heads. It appeared that in the democratic leadership style, deans had this feature at a higher level than assistant Dean / director and the head of department; and assistant dean /director had a higher level than the head of department. In the bureaucratic leadership, deans and department heads seem to have a higher level than assistant deans / directors.

It seemed that the participants' humanistic and charismatic leadership levels did not differ statistically according to marital status, that autocratic, democratic, bureaucratic leadership levels and levels of innovation approaches differed statistically with marital status, and it was observed that these characteristics with significant differences had a higher level of married participants. Given the results obtained, we see that individuals who know how to share, who have the characteristics of being a collective living and a family, are more autocratic, democratic, bureaucratic and innovative. This is because parents need to be autocratic, sometimes democratic and sometimes bureaucratic in their families as a necessity to be a family. It is expected that there will be such a difference due to these reasons. Elkıran [12] found that married academics perceived leadership behaviors a little more than the others in the study of the academicians' views on leadership behaviors in terms of marital status variable. However, Canbolat [8], in his study, revealed that marital status is not an important factor among leadership styles in managerial work.

It is observed that the level of autocratic, democratic and humanist leadership and innovation approach did not differ statistically significantly according to the regular sporting situation that bureaucratic and charismatic leadership levels were statistically different according to the state of regular sports, and the bureaucratic leadership style has resulted in a higher level of non-regular sports participants.

In the charismatic leadership, among the participants who were active in sports, those who participated in sports does sports regularly had a higher level. The high levels of charismatic leadership in academics who regularly participated in sports may be due to the ability of the sports to establish social connections among people, as well as the presence of environments in which the individual may have the opportunity to realize herself. Cheung et al. [9] reported that the charismatic leadership style is the most effective leadership style on the satisfaction of team members in their experimental studies in Hong Hong. It was determined that school administrators showed influential first and second leadership styles, based on the perception and leadership styles of the principals and leaders of physical education and sport at the higher level. Administrators think they have a higher and more significant average than their academics in thinking about adopting transformational and influential leadership behaviors [28]. Charismatic leaders can talk about values, beliefs, propositions, and the importance of trusting each other. These verbal behaviors help to defend new possibilities and harmonize shared proposals. Charismatic leaders show confidence, determination and strong belief in their beliefs, values and ideals. It is expressed that these behaviors of the charismatic leaders are related to the formation of positive affirmation about themselves [27].

It has been observed that the level of autocratic and democratic leadership of the participants did not differ statistically according to the occupational seniority, but the level of humanist, bureaucratic and charismatic leadership 
and the level of innovation approaches differed statistically. In the humanist leadership style, participants with vocational seniority of 6-10 years, $11-15$ years, $16-20$ years and $20+$ years had a higher level than participants with vocational seniority of 1-5 years, and participants with vocational seniority $16-20$ years had this leadership style higher than those with 6-10 years, 11-15 years and 20+ years. In the bureaucratic leadership style, the participants with occupational seniority of 6-10 years, 11-15 years, 16-20 years, and $20+$ years had a higher level than the participants with occupational seniority of 1-5 years, and participants with $20+$ years of vocational seniority had a higher level than those with vocational seniority of 6-10 years, 11-15 years and 16-20 years. In the charismatic leadership style, the participants who had vocational seniority of 6-10 years had higher levels than those who had 1-5 years, $6-10$ years, $11-15$ years and $16-20$ years. In innovation approaches, the participants with 11-15 years, 16-20 years and 20+ years of vocational seniority had a higher level than those with 1-5 years, and the participants with vocational seniority of 11-15 years and $20+$ years had a higher level than the participants with 6-10 years. In Yaşar's [30] study, as the seniority year increased, the leadership perception changed in the negative direction Elkıran [12], in the study of academicians' views on leadership behaviors in terms of seniority variable, showed that leadership behaviors were also improved as seniority increases when the general average scores were examined. It was found that the average of leadership behaviors did not differ by age and work experience, and that the highest average of leadership belonged to those with 11 to 15 years of work experience [33].

It can be seen that there was a significant positive and moderate relation between the participants' levels of democratic leadership and innovation approaches, that there is a positive and moderately significant relationship between levels of autocratic and humanist leadership and innovation approaches, and a positive and moderate relation between bureaucratic leadership levels and innovation approach levels, There appears to be a positive and low level of meaningful relationship between charismatic leadership levels and innovation approach levels.

\section{REFERENCES}

[1] Badarocco, JL. \& Ellsworth, RR. (1978). Leadership and the Quest for Integrity. Harward Busines School Pres. Boston, Massachusetts.

[2] Baltaş. Z. (2011). Kurum içi koçluk. Remzi Kitabevi: İstanbul.

[3] Bayram, Ş. (2013). Liderlik Kavramı ve Liderlik Türlerinin İnovasyon Üzerindeki Etkileri. Gebze Yüksek Teknoloji Sosyal Bilimler Enstitüsü, Yüksek Lisans Tezi, Gebze.
[4] Bentley, T. (2008). Innovating To Learn, Learning To Innovate, Chapter 9 Open Learning A Systems-driven Model of Innovation for Education; OECD Centre for Educational Research and Innovation, OECD Publishing.

[5] Berberoğlu, G. Besler, S. \& Tonus, HZ. (1998). Örgüt Kültürü: Anadolu Üniversitesi İktisadi ve İdari Bilimler Fakültesi Örgüt Kültürü Araştırması. Anadolu Üniversitesi. İktisadi ve İdari Bilimler Fakültesi Dergisi, 14(1-2):29-52.

[6] Brooks, I. (2006). Organisational behaviour: individuals, groups and organisation, Third Edition, Harlow: Pearson Education.

[7] Büyükçolak, K. (1997). Bilgi Çağında Liderlik. 21. Yüzyılda Liderlik Sempozyumu. (5-6 Haziran 1997). Bildiriler Kitabı. Tuzla-İstanbul: Deniz Harp Okulu Basımevi.

[8] Canbolat, SG. (2016). Yöneticilerin Liderlik Tarzlarına İlişkin Çalışan Algıları, Çedaş Grup Şirketleri Örneği. Yüksek Lisans, Sosyal Bilimler Enstitüsü, Hitit Üniversitesi.

[9] Cheung, SO. Thomas, S. Lam, KC. \& Yue, WM. (2001). A Satisfying Leadership Behavior Model for Design Consultants. International Journal of Project Management, 19 (7):421- 429.

[10] Çelik, V. (2007). Akademik Liderlik. XVI. Ulusal Eğitim Bilimleri Kurultayı, Tokat, 216-223.

[11] Dimock, VB. \& McGree, K. (1995). Leading change from the classroom: teachers as leaders, SDL Issues about Change, 4 (4). http://www.sedl.org/change/Issues/Issues44. html (30.05.2006).

[12] Elkıran, ME. (2017). Akademisyenlerin Algıladıkları Yıldırma (Mobbing) Davranışları ile Yöneticilerin Liderlik Davranışları Arasındaki İlişki. Yüksek Lisans Tezi, Eğitim Bilimleri Enstitüsü, Çanakkale Onsekiz Mart Üniversitesi.

[13] Hacıfazlığlu, Ö. (2010). Balance in Academic Leadership: Voices of Women from Turkey and the U.S., Journal of Perspectives in Education, 28(2): 51-62.

[14] Haysom, J. \& Sutton, C. (1973). Innovation in Teacher Education (Science Teacher Education Project). McGraw Hill Higher Education.

[15] Haysom, J. \& Sutton, C. (1974). Theory into Practice. Activities in School for Student Teachers, London: McGraw Hill.

[16] Karasar, N. (2004). Bilimsel Araştırma Yöntemi, Kavramlar, İlkeler, Teknikler. (Beşinci Basım) Ankara, 3A Araştırma Eğitim Danışmanlık.

[17] Korkut, H. (2002). Sorgulanan Yükseköğretim. Ankara: Anı Yayıncilik.

[18] Looney J. W. (2009). Assessment and Innovation in Education. OECD Education Working Papers, No. 24, OECD Publishing. http://dx.doi.org/10.1787/22281454307 3 .

[19] Lubienski C. (2009). Do Quasi-markets Foster Innovation in Education?: A Comparative Perspective, OECD Education Working Papers, No. 25, OECD Publishing. http://dx.doi.org/10.1787/221583463325.

[20] [20] Mcalearney, A.S. (2005) Exploring Mentoring and 
Leadership Development in Health Care Organizations, Career Development International, Vol: 10, No: 6/7, 493-511, 2005.

[21] Moller, G. (1999). You have to want to do this job. Journal of Staff Development, 20 (4). http://www.nsdc.org/library/p ublications/jsd/voices204.cfm (21.06.2006).

[22] NASSP- The Effective Principal A Researc Summary, Virginia, 1992. s:27.

[23] Özkanl1, Ö. ve White, K. (2009). Gender and Leadership in Turkish and Australian Universities, Journal of Equal Opportunities International, 28(4): 324-335.

[24] Ribbins, P. (1997). Leader and Leadership in the School, College and University. London. Cassel.

[25] Sari, I., Soyer, F., \& Gülle, M. (2014). The role of perceived leadership behaviors as predictor of assertiveness levels in individual sport athletes. Procedia-Social and Behavioral Sciences, 152, 446-450.

[26] Solok, S. (1997). Liderlik ve Bir Liderde Bulunmas1 Gereken Özellikler. 21. Yüzyılda Liderlik Sempozyumu. (5 - 6 Haziran 1997). Bildiriler Kitabı. İstanbul. Deniz Harp Okulu basımevi, 2, $404-406$.

[27] Sosik, JJ. \& Dworakivsky, AC. (1998). Self-Concept Based Aspects of The Charismatic Leader: More Than Meets The Eye, Leadership Quarterely, 9 (4) 503-526.

[28] Şirin, E. F., \& Yetim, A. A. (2009) Beden Eğitimi ve Spor Yüksekokulu Yöneticilerinin Dönüşümcü ve Etkileşimci Liderlik Stillerinin İncelenmesi. Gazi Beden Eğitimi ve Spor Bilimleri Dergisi, 13(4), 31-46.

[29] Şişman, M. (2007). Örgütler ve Kültürler. Ankara: Pegem A Yayıncilik.

[30] Yaşar, H. (2015). Akademisyenlerin Hizmetkar Liderlik Algıları (Harran Universitesi Orneği). Bozok Universitesi İlahiyat Fakultesi Dergisi, 7 (7), 159-170.

[31] Yielder, J. \& Codling, A. (2004). Management and leadership in the contemporary university. Journal of Higher Education Policy and Management, 26(3), 315-328.

[32] Yukl, G. A. (2013). Leadership in organizations. Pearson Education India.

[33] Yücel, İ., \& Akgül, İ. (2016) Liderlik Stilleri ile Örgütsel Bağlılık Arasındaki İlişskide İş Tatmininin Aracılık Etkisi: Akademisyenler Üzerine Bir Çalışma. Cumhuriyet Üniversitesi İktisadi ve İdari Bilimler Dergisi, 17(1), 209-226. 